\title{
Chapter 81 \\ Report on the Global Environment \\ Competitiveness of Latvia
}

Latvia is located in the west of the plain in Eastern Europe, near the east coast of Baltic sea, and borders on Estonia, Russia, Belarus and Lithuania. The climate is temperate broad-leaved forest climate, and the peat resources are rich. It covers 62 thousand square kilometres. It had a population of 2.1 million and domestic production the gross (GDP) of USD 28 billion in 2011. Through the evaluation of global environment competitiveness, we can know that the environment competitiveness index of Latvia ranks at 36 in 133 countries.

Fig. 81.1 Contribution of

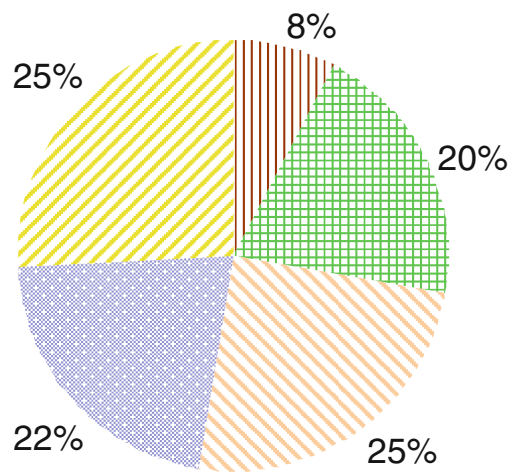

Resource Environment Competitiveness

\# Ecological Environment Competitiveness

Environment Carrying Competitiveness

Environment Management Competitiveness sub-index of GEC

Environment Harmony Competitiveness 


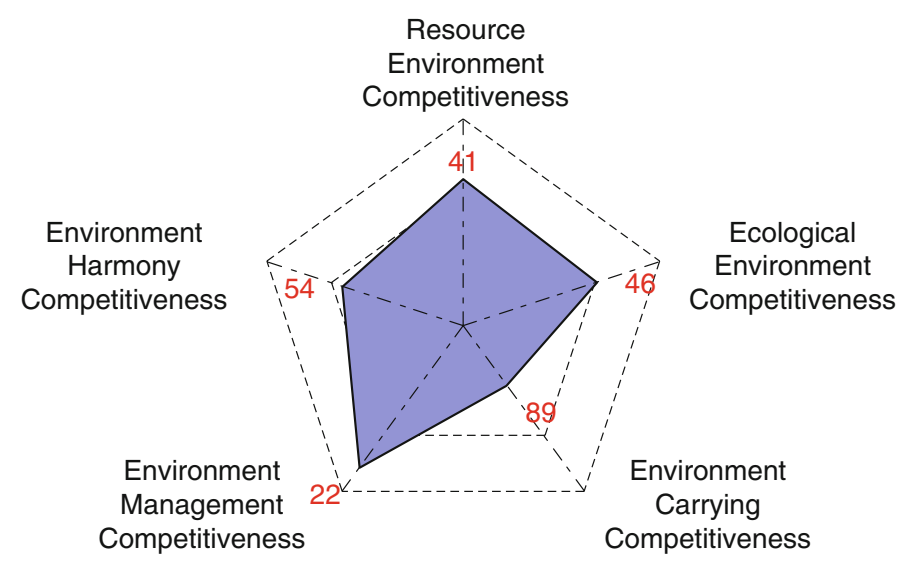

Fig. 81.2 Rank of sub-index of GEC

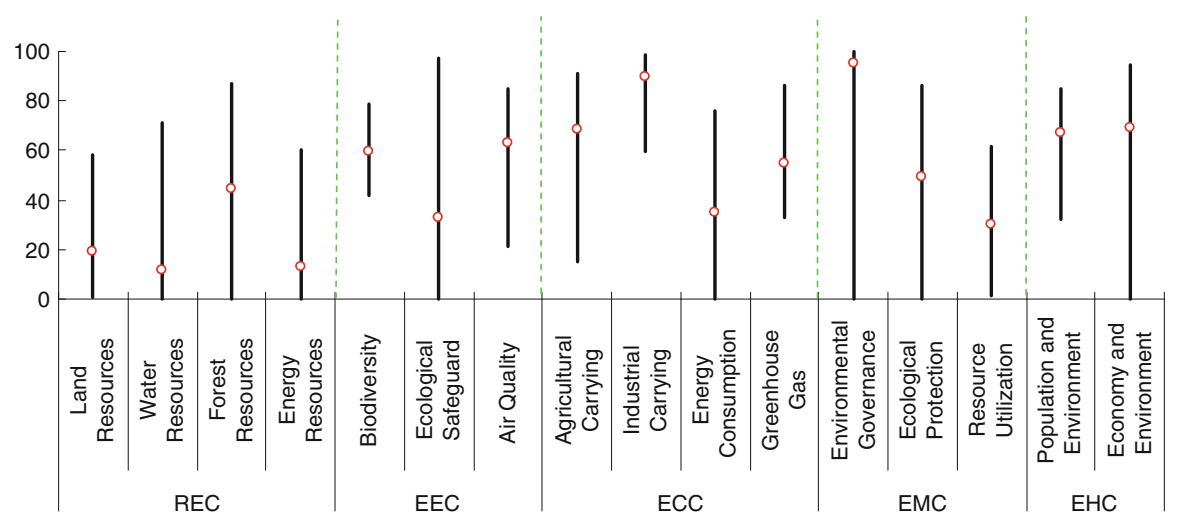

Fig. 81.3 Score and rank of the pillars of GEC

Table 81.1 Score and rank of all indicators of GEC

\begin{tabular}{|c|c|c|c|c|c|}
\hline Indicators & Score & Rank & Indicators & Score & Rank \\
\hline $\begin{array}{l}1 \text { Resource Environment } \\
\text { Competitiveness }\end{array}$ & 20.30 & 41 & $\begin{array}{l}\text { Total internal renewable } \\
\text { water resources }\end{array}$ & 13.58 & 67 \\
\hline 1.1 Land Resources & 19.52 & 32 & 1.3 Forest Resources & 44.34 & 19 \\
\hline Land area per capita & 5.42 & 36 & Growing stock in forest and & 51.92 & 53 \\
\hline Percentage of arable land & 31.49 & 43 & other wooded land & & \\
\hline $\begin{array}{l}\text { to total land area } \\
\text { Arable land per capita }\end{array}$ & 26.34 & 11 & $\begin{array}{l}\text { Proportion of land area } \\
\text { covered by forest }\end{array}$ & 63.37 & 12 \\
\hline 1.2 Water Resources & 11.82 & 79 & Forest area per capita & 11.40 & 20 \\
\hline Surface water & 6.12 & 53 & 1.4 Energy Resources & 13.27 & 35 \\
\hline Annual precipitation & 22.54 & 82 & Fossil energy & 0.00 & 64 \\
\hline Groundwater & 5.06 & 87 & Energy production & 0.95 & 63 \\
\hline
\end{tabular}


Table 81.1 (continued)

\begin{tabular}{|c|c|c|c|c|c|}
\hline Indicators & Score & Rank & Indicators & Score & Rank \\
\hline $\begin{array}{l}\text { Proportion of combustible } \\
\text { renewables and waste to }\end{array}$ & 60.20 & 20 & $\begin{array}{l}\text { Ratio of clean energy } \\
\text { consumption }\end{array}$ & 12.20 & 46 \\
\hline $\begin{array}{l}\text { total energy consumption } \\
\text { Net energy imports of the }\end{array}$ & 5.99 & 93 & $\begin{array}{l}\text { Elasticity of energy } \\
\text { consumption }\end{array}$ & 14.80 & 22 \\
\hline $\begin{array}{c}\text { energy consumption } \\
2 \text { Ecological Environment }\end{array}$ & 52.69 & 46 & $\begin{array}{l}\text { Elasticity of electric power } \\
\text { consumption }\end{array}$ & 13.47 & 20 \\
\hline Competitiveness & & & 3.4 Greenhouse Gas & 54.63 & 114 \\
\hline $\begin{array}{l}2.1 \text { Biodiversity } \\
\text { Threatened fish species }\end{array}$ & $\begin{array}{l}59.33 \\
97.17\end{array}$ & $\begin{array}{l}29 \\
17\end{array}$ & $\begin{array}{l}\text { Growth rate of } \mathrm{CO}_{2} \\
\text { emissions }\end{array}$ & 30.95 & 119 \\
\hline $\begin{array}{l}\text { Threatened mammal species } \\
\text { Threatened plant species }\end{array}$ & $\begin{array}{r}99.46 \\
100.00\end{array}$ & $\begin{array}{l}2 \\
1\end{array}$ & $\begin{array}{l}\text { Growth rate of Methane } \\
\text { emissions }\end{array}$ & 59.57 & 60 \\
\hline $\begin{array}{l}\text { GEF benefits index for } \\
\text { biodiversity }\end{array}$ & 0.00 & 128 & $\begin{array}{l}\mathrm{CO}_{2} \text { emissions per unit } \\
\text { of land area }\end{array}$ & 99.86 & 60 \\
\hline $\begin{array}{l}\text { 2.2 Ecological Safeguard } \\
\text { Terrestrial protected areas }\end{array}$ & $\begin{array}{l}32.69 \\
48.64\end{array}$ & $\begin{array}{l}37 \\
35\end{array}$ & $\begin{array}{l}\mathrm{CO}_{2} \text { emissions per unit } \\
\text { of energy consumption }\end{array}$ & 51.83 & 45 \\
\hline $\begin{array}{l}\text { Marine protected areas } \\
\text { 2.3 Air Ouality }\end{array}$ & $\begin{array}{r}8.76 \\
62.73\end{array}$ & $\begin{array}{l}29 \\
56\end{array}$ & $\begin{array}{l}4 \text { Environment Management } \\
\text { Competitiveness }\end{array}$ & 57.29 & 22 \\
\hline $\begin{array}{l}\text { Inhalable particles (PM10) } \\
\text { Particulate matter (PM2 5) }\end{array}$ & $\begin{array}{l}91.24 \\
90.88\end{array}$ & $\begin{array}{r}9 \\
31\end{array}$ & $\begin{array}{l}\text { 4.1 Environmental } \\
\text { Governance }\end{array}$ & 94.99 & 45 \\
\hline Index of indoor air pollution & $\begin{array}{l}90.88 \\
32.80\end{array}$ & 62 & $\begin{array}{l}\text { Agricultural chemicals } \\
\text { regulation }\end{array}$ & 90.48 & 37 \\
\hline $\begin{array}{l}\text { Nitrogen oxides emission } \\
\text { Sulfur dioxide emission }\end{array}$ & $\begin{array}{l}68.75 \\
40.99\end{array}$ & $\begin{array}{r}26 \\
4\end{array}$ & $\begin{array}{l}\text { regulation } \\
\text { Percentage of the rural }\end{array}$ & 96.00 & 46 \\
\hline $\begin{array}{l}3 \text { Environment Carrying } \\
\text { Competitiveness }\end{array}$ & 65.54 & 89 & $\begin{array}{l}\text { population with access } \\
\text { to an improved water } \\
\text { source }\end{array}$ & & \\
\hline 3.1 Agricultural Carrying & 68.77 & 53 & Percentage of the urban & 100.00 & 1 \\
\hline $\begin{array}{l}\text { Cereal yield per unit of } \\
\text { arable land }\end{array}$ & 25.90 & 75 & $\begin{array}{l}\text { population with access } \\
\text { to an improved water }\end{array}$ & & \\
\hline $\begin{array}{l}\text { Fertilizer consumption } \\
\text { per unit of arable land }\end{array}$ & 94.76 & 65 & $\begin{array}{l}\text { source } \\
\text { 4.2 Ecological Protection }\end{array}$ & 49.42 & 16 \\
\hline $\begin{array}{l}\text { Annual freshwater } \\
\text { withdrawals for } \\
\text { agriculture per unit of }\end{array}$ & 99.94 & 17 & $\begin{array}{l}\text { Area of plantation and } \\
\text { afforestation } \\
\text { Biome protect }\end{array}$ & 0.81 & 44 \\
\hline $\begin{array}{l}\text { arable land } \\
\text { 3.2 Industrial Carrying }\end{array}$ & 89.88 & 83 & $\begin{array}{l}\text { Overfishing of fishing } \\
\text { resources }\end{array}$ & 63.64 & 29 \\
\hline $\begin{array}{l}\text { Net exports as a percentage } \\
\text { of GDP }\end{array}$ & 73.34 & 94 & 4.3 Resource Utilization & 30.09 & 99 \\
\hline Electric power consumption & 90.23 & 82 & $\begin{array}{l}\text { Utilization rate of water } \\
\text { resources }\end{array}$ & 0.04 & 112 \\
\hline $\begin{array}{l}\text { per unit of value added } \\
\text { of industry }\end{array}$ & & & $\begin{array}{l}\text { Percentage of total internal } \\
\text { renewable water }\end{array}$ & 44.70 & 92 \\
\hline $\begin{array}{l}\mathrm{SO}_{2} \text { emissions per unit of } \\
\text { value added of industry }\end{array}$ & 99.98 & 14 & $\begin{array}{l}\text { resources to total water } \\
\text { resources }\end{array}$ & & \\
\hline $\begin{array}{l}\text { Annual freshwater } \\
\text { withdrawals for industry }\end{array}$ & 95.96 & 83 & $\begin{array}{l}\text { Percentage of agricultural } \\
\text { land to total land area }\end{array}$ & 34.84 & 103 \\
\hline $\begin{array}{l}\text { per value added of } \\
\text { industry }\end{array}$ & & & Percentage of fossil fuel & 40.78 & 41 \\
\hline 3.3 Energy Consumption & 35.08 & 46 & $\begin{array}{l}\text { to total energy } \\
\text { tolit }\end{array}$ & & \\
\hline $\begin{array}{l}\text { Energy consumption per } \\
\text { unit of land area }\end{array}$ & 99.85 & 50 & consumption & & \\
\hline
\end{tabular}


Table 81.1 (continued)

\begin{tabular}{|c|c|c|c|c|c|}
\hline Indicators & Score & Rank & Indicators & Score & Rank \\
\hline $\begin{array}{l}5 \text { Environment Harmony } \\
\text { Competitiveness }\end{array}$ & 68.28 & 54 & $\begin{array}{l}\mathrm{CO}_{2} \text { emissions (metric tons } \\
\text { per capita) }\end{array}$ & 90.34 & 72 \\
\hline $\begin{array}{l}\text { 5.1 Population and } \\
\text { Environment }\end{array}$ & 67.08 & 86 & $\begin{array}{l}\text { Energy consumption } \\
\text { per capita }\end{array}$ & 85.55 & 76 \\
\hline $\begin{array}{l}\text { Improved sanitation } \\
\text { facilities (\% of }\end{array}$ & 78.00 & 80 & $\begin{array}{l}5.2 \text { Economy and } \\
\text { Environment }\end{array}$ & 69.48 & 32 \\
\hline $\begin{array}{l}\text { population with access) } \\
\text { Motor vehicles (per } 1,000\end{array}$ & 43.58 & 104 & $\begin{array}{l}\text { Land resource utilization } \\
\text { efficiency }\end{array}$ & 0.13 & 58 \\
\hline $\begin{array}{c}\text { people) } \\
\text { Renewable internal }\end{array}$ & 9.84 & 41 & $\begin{array}{l}\text { Sulfur dioxide emissions } \\
\text { per unit of GDP }\end{array}$ & 99.62 & 10 \\
\hline $\begin{array}{l}\text { freshwater resources } \\
\text { per capita }\end{array}$ & & & $\begin{array}{l}\text { Carbon dioxide emissions } \\
\text { per unit of GDP }\end{array}$ & 89.14 & 60 \\
\hline $\begin{array}{l}\mathrm{SO}_{2} \text { emissions (metric tons } \\
\text { per capita) }\end{array}$ & 99.35 & 19 & $\begin{array}{l}\text { Energy consumption } \\
\text { per unit of GDP }\end{array}$ & 89.04 & 48 \\
\hline
\end{tabular}

Table 81.2 Rank distribution of the individual indicators of GEC

\begin{tabular}{lllllll}
\hline & $\begin{array}{l}\text { Number } \\
\text { of the } \\
\text { individual } \\
\text { indicators }\end{array}$ & $\begin{array}{l}\text { Rank } \\
1-10\end{array}$ & $\begin{array}{l}\text { Rank } \\
11-30\end{array}$ & $\begin{array}{l}\text { Rank } \\
31-60\end{array}$ & $\begin{array}{l}\text { Rank } \\
61-100\end{array}$ & $\begin{array}{l}\text { Rank } \\
101-133\end{array}$ \\
\hline Sub-index & 14 & 0 & 3 & 6 & 5 & 0 \\
\hline $\begin{array}{l}\text { Resource Environment } \\
\quad \text { Competitiveness } \\
\text { Ecological Environment } \\
\quad \text { Competitiveness }\end{array}$ & 11 & 3 & 3 & 4 & 1 & 0 \\
$\begin{array}{c}\text { Environment Carrying } \\
\quad \text { Competitiveness }\end{array}$ & 15 & 0 & 2 & 6 & 5 & 2 \\
$\begin{array}{c}\text { Environment Management } \\
\quad \text { Competitiveness }\end{array}$ & 10 & 1 & 1 & 4 & 2 & 2 \\
$\begin{array}{c}\text { Environment Harmony } \\
\quad \text { Competitiveness }\end{array}$ & 10 & 1 & 1 & 4 & 3 & 1 \\
Total & 60 & 5 & 10 & 24 & 16 & 5 \\
\hline
\end{tabular}

Open Access This chapter is distributed under the terms of the Creative Commons Attribution Noncommercial License, which permits any noncommercial use, distribution, and reproduction in any medium, provided the original author(s) and source are credited. 